\title{
CROWDSOURCING AUXILIANDO MÉTODOS DE PESQUISA: O EXCEDENTE COGNITIVO EM PROL DA PRODUÇÃo CIENTífica
}

\author{
CROWDSOURCING AIDING RESEARCH METHODS: \\ COGNITIVE SURPLUS IN FAVOR OF SCIENTIFIC PRODUCTION
}

\section{RESUMO}

As tecnologias de informação e comunicação vem remodelando os processos de interação humana, tornando-os mais efetivos e acelerados. Neste texto, discute-se o crowdsourcing como uma ferramenta de método de pesquisa. Para tanto, apresenta-se uma breve fundamentação teórica a respeito da produção e metodologia científicas consequentes da influência das tecnologias. $\mathrm{O}$ artigo não tem a pretensão de propor um método plenamente novo com as características do crowdsourcing, mas sim introduzir uma discussão necessária sobre o excedente cognitivo que ainda não é utilizado no campo científico e assim, apresentar esta técnica como ferramenta para os atuais métodos científicos.

PAlaVRAS-CHAVE: Crowdsourcing. Metodologia científica. Comunicação científica. Mídias sociais. Cultura da participação.

\begin{abstract}
The information and communication technologies is remodeling the processes of human interaction, making them more effective and accelerated. In this article, the crowdsourcing is presented as search method. To it, presents a brief theoretical foundation about the scientific production and methodology consequent of the influence of technology. This article have not the pretension to propose a method with crowdsourcing's characteristics, but yes introduce a needed discussion about cognitive surplus that is not use in the scientific field yet to present this technique as scientific method.
\end{abstract}

KEYWORDS: Crowdsourcing. Scientific methodology. Scientific communication. Social media. Culture of participation.

\section{INTRODUÇÃO}

Por muito tempo a oralidade foi a única forma de se transmitir informação entre as gerações. As histórias e formas de fazer qualquer atividade - doméstica, de caça, de fabricação, de sociabilidade - eram passadas de pai para filho, de mestre para discípulo, de líder para liderado e era o que bastava. A prática e suas consequentes melhorias eram a própria forma de manter a informação, mesmo com a escrita rupestre.

Com o avanço da escrita e a contribuição da imprensa, a informação tornou-se muito mais simples de ser disseminada, o que garantiu dos indivíduos uma vontade muito maior de conhecer e entender as coisas e os fenômenos, mesmo que tenhamos o fato da elitização dos livros em tempos medievais (BURKE, 2012). É esta vontade de conhecer com uma gama de

\footnotetext{
${ }^{1}$ Mestrando no Programa de Pós-graduação em Gestão da Informação da Universidade do Estado de Santa Catarina (UDESC). E-mail: jorge.exlibris@gmail.com

Recebido em: 26/02/2015 - Aceito em: 27/04/2015
} 
necessidades que precisavam ser preenchidas que moveu as invenções e o desenvolvimento da sociedade de forma plena.

Para garantir e facilitar a continuidade destes processos inventivos, atribui-se a Aristóteles o início do método científico, tentando formatar como se pensa, cria e reproduz o conhecimento a partir do processo de indução e dedução (VICENTE, 2008). Entretanto, muitos séculos depois, é com Descartes e seu "Discurso do Método" (1596-1650) "que foram lançados, de fato, os fundamentos do método científico moderno" (PROFICIÊNCIA, 2014).

O processo de pesquisar delineado em formato de método é uma ferramenta bastante importante, pois além de colaborar para o desenvolvimento de qualquer pesquisa, ele galvaniza a ciência. O método científico é uma forma também de garantir que todos possam utilizar uma mesma linguagem, além de servir como um norte para outros indivíduos a obter resultados de forma semelhante. Outrossim, ela permite entender melhor os resultados de uma pesquisa, bem como suas limitações.

Com o rápido avanço e adoção por parte da sociedade das tecnologias da informação e comunicação (TICs), muitos processos foram criados ou atualizados e tornaram-se mais rápidos em diversas áreas de atuação profissional e do conhecimento. No campo da metodologia científica, as tecnologias vieram mais como ferramentas de auxílio, ainda não propondo uma metodologia propriamente dita baseada em tecnologias.

Um destes processos que foram criados com as possibilidades das TICs é o do crowdsourcing, que se atribui do excedente cognitivo das pessoas conectadas em rede a fim de solucionar alguma questão. Ele é apresentado neste artigo de forma a introduzir questões relevantes para, por fim, representa-lo como uma técnica que contribua para os atuais métodos científicos.

\section{PRODUÇÃO CIENTÍFICA E MÉTODOS CIENTÍFICOS}

A produção científica, de conhecimento, no formato como se conhece hoje, tem início reconhecido no século XVIII. Burke (2012, p. 22) informa que neste período a palavra research (pesquisa) "passou a ser cada vez mais frequente nos títulos de livros [...], para descrever investigações em uma variedade de campos intelectuais".

Para a produção do conhecimento, torna-se necessário delimitar algumas características do ato de pesquisar que posteriormente se padronizam nos métodos científicos. Silva (2001) delineia um breve histórico dos métodos científicos pioneiros: Século XV: senso comum e aplicação religiosa orientam as investigações humanas; Século XVI: método experimental apresentado por Galileu, método indutivo por Francis Bacon e o dedutivo por René Descartes; Século XVII: contribuições de Isaac Newton a partir das reflexões propostas por Bacon e Galileu fortalecendo o método experimental.

Todas estas propostas encaixam-se na definição de "método" proposta por Lakatos e Marconi (2010, p. 83): 
O método é o conjunto das atividades sistemáticas e racionais que, com maior segurança e economia, permite alcançar o objetivo - conhecimentos válidos e verdadeiros -, traçando o caminho a ser seguido, detectando erros e auxiliando as decisões do cientista.

Além de contribuir para o processo de pesquisa, os métodos são uma forma de linguagem técnica internacional, facilitando o entendimento dos indivíduos perante o intuito do pesquisador.

\section{SOCIEDADE EM REDE E A CULTURA DA PARTICIPAÇÃO}

Cada momento histórico é demarcado por fatores sociais, econômicos e culturais que ditam o cotidiano das pessoas, o modus operandi de determinada realidade. Estes fatores vão desde aparatos tecnológicos que facilitam a vida dos indivíduos, até a linguagem e comunicação.

Durante os últimos anos, a tecnologia da informação tem se demonstrando uma ferramenta primordial para a composição de uma nova sociedade, pois decorre de uma revolução que se infiltra em todas as esferas da atividade humana (CASTELLS, 2010). Outras possibilidades são criadas com o aporte deste novo contexto com a finalidade de facilitar e acelerar processos que em outro tempo demoravam demasiadamente. Castells (2010, p. 43) nos apresenta o início desta revolução:

[...] quando na década de 1970 um novo paradigma tecnológico, organizado com base na tecnologia da informação, veio a ser constituído, principalmente nos Estados Unidos, foi um segmento específico da sociedade norte-americana, em interação com a economia global e a geopolítica mundial, que concretizou um novo estilo de produção, comunicação, gerenciamento e vida.

É justamente dentro deste contexto militar norte-americano que surge a principal ferramenta proveniente desta revolução social com o estopim na tecnologia da informação, que é a Internet.

Nos últimos 20 anos, quando a Internet é assumida como um espaço legítimo de troca de informações, com diferentes interesses e necessidades, a sociedade passa a ser vista como que estando em rede. Relações de troca são estabelecidas de modo a torná-la um ambiente onde quase tudo pode ser feito, interligando indivíduos com iguais ou semelhantes interesses. Dias (2013) afirma que esta sociedade transcende o tempo e o espaço dentro de uma dimensão virtual impulsionada pelas novas tecnologias.

Com a aceitação das tecnologias pela sociedade e mais a sua democratização (desde o barateamento de dispositivos tecnológicos até a criação de leis a respeito do acesso à informação), "uma série de acontecimentos [...] tem alterado significativamente a organização dos sistemas sociais, políticos e econômicos em âmbito mundial" (CORRÊA, 
2004, p. 1). Isto decorre também, não meramente, da participação em rede entre os indivíduos envolvidos.

Para entender esta questão da participação para a concretização de uma sociedade em rede, é necessário apresentar a questão do excedente cognitivo. Shirky (2011, p. 15) é factual ao afirmar que "[...] podemos agora tratar o tempo livre como um bem social geral que pode ser aplicado a grandes projetos criados coletivamente, em vez de um conjunto de minutos individuais a serem aproveitados por uma pessoa de cada vez".

O excedente cognitivo, portanto, é este tempo livre de cidadãos escolarizados em prol de um objetivo maior e coletivo. O desenvolvimento de alguns softwares é tido como o marco inicial do uso do excedente cognitivo de forma bem sucedida, como foi o caso do Linux (SHIRKY, 2011). Há também a presença do excedente cognitivo em produtos e serviços de entretenimento, em sua maioria, como YouTube, Napster e o mais famoso deles, a Wikipedia.

A Wikipedia é um projeto para produzir uma enciclopédia de conteúdo livre que pode ser editada por todos. Começou, formalmente, em 15 de janeiro de 2001, como um complemento ao seu similar, o projeto Nupedia (escrito por especialistas). Ele acabou substituindo a Nupedia, crescendo até se tornar um projeto de amplitude global. (WIKIPEDIA, 2014).

Esta participação não se estabelece por meio de trocas (não há um pagamento financeiro, por exemplo), o benefício é intrínseco a toda sociedade pelo fato dela manter-se informada.

Outros exemplos de excedente cognitivo são apresentados nas obras de Israel (2010) e Giardelli (2012), mas com uma finalidade social. Israel, especificamente, trata do uso da mídia social Twitter em projetos para a educação, política, saúde e mesmo dentro do ativismo político. Já Giardelli (2012) apresenta as ferramentas tecnológicas auxiliando como um todo a realidade humana, com a gestão coletiva e participativa e o financiamento coletivo a partir de aplicativos em dispositivos móveis.

A efetividade, confiabilidade e respeitabilidade destes projetos participativos não será tratada dentro deste artigo, mas alguns autores já refletiram a respeito, como Weinberger (2007) ao afirmar que o jornal Nature's descobriu que os artigos escritos dentro da Wikipedia têm uma precisão bastante próxima do que é escrito na famosa enciclopédia Britannica.

Desta cultura da participação, pode-se extrair um valor que, conforme Shirky (2011, p. 144), “[...] jamais foi imaginado, graças ao aperfeiçoamento de nossa habilidade de nos conectarmos uns aos outros e de nossa imaginação do que será possível". Giardelli (2012, p. 58) ainda complementa que "a partir de agora, sua empresa calculará não apenas o return over investiment (ROI), mas o return over attention (ROA)”, ou seja, esta participação será efetiva dentro das organizações. 
Neste contexto de uma sociedade em rede com uma cultura participativa, alguns modelos foram criados ou remodelados. O tópico a seguir abordará um modelo de participação coletiva como método de pesquisa.

\section{CROWDSOURCING AUXILIANDO OS MÉTODOS DE PESQUISA}

Continuando com a reflexão de que as tecnologias vêm remodelando ou beneficiando alguns processos da realidade humana, pensa-se que ela também poderá surtir algum efeito na forma de pesquisar, mais propriamente no desenvolvimento da produção científica.

Neste contexto, uma tendência é como aproveitar a "sabedoria das multidões", pois conforme Pisani e Piotet (2010, p. 268), "a participação é de fato o desafio mais sério que as mídias devem enfrentar. Ela recoloca em questão sua autoridade e seu papel social”, ou seja, as mídias conseguem atribuir novas identidades aos indivíduos, diferente do que são em mundo real.

Num agrupamento de tecnologias com mídias sociais, alguns modelos foram criados para acelerar processos ou mesmo trazendo uma nova roupagem aos já tradicionais. Um destes modelos é o crowdsourcing, que Seltzer e Mahmoudi (2013, p. 6. Tradução livre) nos elucidam:

O termo "crowdsourcing" é usualmente identificado a partir de uma série de artigos escritos por Jeffrey Howe na revista Wired (2006) e seu livro publicado em 2009. Em um de seus artigos de 2007, Howe relata a ascensão de uma contracorrente que ele identificou para a terceirização de problemas em indústrias da Índia e da China.

Além destes problemas industriais, Howe também demonstra o poder da colaboração dos indivíduos em projetos de grandes empresas do entretenimento, setor alimentício e saúde (HOWE, 2006), o que confirma a amplitude das tecnologias.

O modelo de crowdsourcing visa convidar indivíduos que estão conectados à rede para trabalhar, de forma coletiva, a solução de algum problema, independente de espaço e tempo. Arolas e González (2012) confirmam:

[o crowdsourcing] é um tipo de atividade online participativa em que o indivíduo, uma instituição, uma organização sem fins lucrativos [...] propõe a um grupo de indivíduos de diferentes conhecimentos, heterogeneidade e número, através de uma chamada flexível e aberta, o compromisso voluntário de uma tarefa.

Neste molde pode-se citar o case de Harvard, que em 2010 mobilizou pesquisadores que estudavam o tipo 1 do diabetes a avançarem nos estudos (OLIVEIRA, 2012) ou então o livro de Israel (2010) que foi todo escrito com a colaboração de seus seguidores no Twitter.

Os melhores ambientes para crowdsourcing são as próprias mídias sociais, justamente pela heterogeneidade apontada por Arolas e González (2012), entretanto, ferramentas online foram criadas especificamente para este propósito. O site Inc (20??) apresenta algumas, como 
Kickstarter $^{2}$, MyGenGo $^{3}$, Quora ${ }^{4}$ e GetSatisfaction ${ }^{5}$. Já no campo científico, ainda há certa carência de espaços próprios para isso.

O que se permite observar é que as mídias sociais assumem um papel importante para o desenvolvimento do crowdsourcing científico, pois em blogs, Twitter e Facebook ${ }^{6}$ os próprios cientistas se comunicam com o seu público para a discussão de um tema, mesmo que ainda de forma "tímida". Alisson (2014) afirma que o índice de publicação de notícias a respeito do que é produzido cientificamente é bastante alto, entretanto, falta ainda dos cientistas uma forma de engajar o público para a produção coletiva.

Se as mídias sociais e o excedente cognitivo estão remodelando as atividades humanas, conforme explicitado e fundamentado, torna-se necessário um olhar mais atento para a produção científica. Uma forma de tornar o crowdsourcing como método de pesquisa com etapas de início, meio e fim claramente identificadas - poderia ser um passo inicial para um efetivo desenvolvimento científico coletivo. Entretanto, também é um desafio modelar um processo que depende da boa vontade das pessoas pertencentes a uma rede, mesmo que forte, para chegar a resultados efetivos.

Trabalhar com crowdsourcing também dependerá do monitoramento das opiniões do público nas plataformas que o cientista for utilizar, principalmente se for nas mídias sociais. Silva (2012) destaca que as mídias sociais têm suas particularidades, pois dependem de uma manifestação cultural em tempo real, tornando assim o monitoramento uma atividade imprescindível. Além disso, também dependerá do cientista compreender como funciona a dinâmica das mídias sociais, pois "à medida que os usuários comentam e replicam informações em conexões de mídias, mais elas crescem em relevância e representatividade na dinâmica associativa das conexões em rede" (ALZAMORA; ZILLER, 2013, p. 124).

Para que o crowdsourcing seja visto como uma ferramenta de auxílio ao método científico, é necessário antes refletir sobre alguns problemas e desafios, de ordem técnica ou mesmo social. Wagorn (2014, tradução livre) apresenta alguns itens: a) Falta de confidencialidade: dependendo de alguns projetos, este item pode se tornar um grande empecilho, pois estando em trabalho coletivo, seu nível de confidencialidade é quase nulo; b) Ruídos de comunicação: a questão da interpretação sobre opiniões de outrem pode também ser uma barreira para o desenvolvimento do projeto; c) Ordem técnica: neste item o autor apresenta alguns problemas de ordem técnica computacional; d) Ideias não são soluções: dependendo do andamento da pesquisa coletiva, o processo pode entrar num círculo se mais ideias surgirem do que soluções para os problemas apresentados ou metas que devem ser alcançadas.

\footnotetext{
${ }^{2}$ https://www.kickstarter.com/ (Acesso em 11 jun. 2014)

${ }^{3} \mathrm{http}: / /$ gengo.com/ (Acesso em 11 jun. 2014)

${ }^{4}$ https://www.quora.com/ (Acesso em 11 jun. 2014)

${ }^{5} \mathrm{https} / /$ getsatisfaction.com/corp/ (Acesso em 11 jun. 2014)

${ }^{6} \mathrm{O}$ engajamento é maior, obviamente, nessas mídias não-científicas, entretanto, também há um crescimento de espaços próprios para a ciência, como Ademia.edu, ResearchGate, Mendeley e outros.
} 
Ainda com Wagorn (2014, tradução livre), apresenta-se algumas soluções para estes problemas: criação de um plano de comunicação claro, trabalhar com equipe multidisciplinar, determinar um rol de atividades a serem desenvolvidas até chegar ao objetivo proposto pelo pesquisador e mesmo tornar a pesquisa interessante, que motive o público engajado.

\section{CONSIDERAÇÕES FINAIS}

As tecnologias da informação e, consequentemente, as mídias sociais, tem se apresentando como inovadoras aliadas para a sociedade, ao mesmo tempo, que também se apresentam como grandes desafios para sua adaptabilidade. Percebe-se ainda um contexto de testes, estudos e descobertas.

O crowdsourcing e mesmo a sabedoria coletiva como um todo, demonstra ser uma técnica merecedora de muito estudo, pois seu potencial é grande e com um grau de inovação que ainda não foi apresentado pelos tradicionais métodos de pesquisa. Com diversos pontos de vista para o pesquisador, o resultado pode trazer uma amplitude muito maior de reflexões.

Se adotado fortemente na produção científica e se consolidado como uma ferramenta que auxilie métodos científicos, será necessário repensar alguns pontos, como o papel dos comitês de ética e as pesquisas com seres humanos, além das questões autorais que são sempre determinantes nas discussões envolvendo mídias sociais.

O novo dentro de alguma área que já é consolidada ainda leva um período de tempo para ser adotado com confiança, que é o caso do crowdsourcing. Considera-se, entretanto, que a produção científica e sua disseminação, não podem descartar as hipóteses de inovação e benefícios proporcionados pelas tecnologias da informação e comunicação.

\section{REFERÊNCIAS}

\section{ALISSON, Elton. Interatividade da internet mudou a forma de comunicar ciência.}

Publicado em: 09 maio 2014. Disponível em: <http://agencia.fapesp.br/19049>. Acesso em: 12 jun. 2014.

ALZAMORA, Geane; ZILLER, Joana. A dinâmica associativa das mídias sociais: semiose e convergência. Teccogs, dossiê, n. 8, jun.-dez. 2013. Disponível em:

<http://www4.pucsp.br/pos/tidd/teccogs/dossies/2013/edicao_8/2-

dinamica_associativa_midias_sociais-semiose_convergencia.pdf $>$. Acesso em: 12 jun. 2014.

AROLAS, Enrique Estellés; GUEVARA, Fernando González Ladrón de. Towards an integrated crowdsourcing definiton. Journal of Information Science, vol. 38, no. 2, April 2012. Disponível em: 〈http://jis.sagepub.com/content/38/2/189.short?rss=1\&ssource=mfr > Acesso em: 11 jun. 2014. 
BURKE, Peter. Uma história social do conhecimento: da enciclopédia à Wikipedia. Rio de Janeiro: Zahar, 2012.

CASTELLS, Manual. A sociedade em rede: volume 1. São Paulo: Paz e Terra, 2010.

CORRÊA, Cynthia Harumy Watanabe. Comunidades virtuais gerando identidades na sociedade em rede. Ciberlegenda, n. 13, 2004. Disponível em:

<http://www.uff.br/ciberlegenda/ojs/index.php/revista/article/view/226>. Acesso em: 09 jun. 2014.

DIAS, Adelaide. Definição de sociedade em rede. Publicado em: 25 fev. 2013. Disponível em: <http://pt.slideshare.net/adelaidedias9/definio-de-sociedade-em-rede>. Acesso em: 09 jun. 2014.

GENGO. Site. Disponível em: <http://gengo.com/>. Acesso em: 11 jun. 2014.

GETSATISFACTION. Site. Disponível em: <https://getsatisfaction.com/corp/>. Acesso em: 11 jun. 2014.

GIARDELLI, Gil. Você é o que você compartilha: e-agora: como aproveitar as oportunidades de vida e trabalho na sociedade em rede. São Paulo: Gente, 2012.

HOWE, Jeffrey. The rise of crowdsourcing. Publicado em jun. 2006. Disponível em: <http://archive.wired.com/wired/archive/14.06/crowds.html?pg=1\&topic=crowds\&topic_set =>. Acesso em: 10 jun. 2014 .

INC. 6 cool crowdsourcing business tools. Publicado em [20??]. Disponível em: <http://www.inc.com/ss/how-use-crowdsourcing-business\#0>. Acesso em: 11 jun. 2014.

ISRAEL, Shel. A era do Twitter: como a ferramenta de mídia colaborativa mais dinâmica da atualidade pode revolucionar seus negócios. Rio de Janeiro: Elsevier, 2010.

KICKSTARTER. Site. Disponível em: <https://www.kickstarter.com/>. Acesso em: 11 jun. 2014.

LAKATOS, Eva Maria; MARCONI, Marina de Andrade. Fundamentos de metodologia científica. 7. ed. São Paulo: Atlas, 2010.

OLIVEIRA, Vivian. $\mathbf{O}$ crowdsourcing à frente da mídia colaborativa e democrática: uma perspectiva cidadã para a web 2.0. Publicado em 2012. Disponível em:

<http://www.unicentro.br/redemc/2012/artigos/34.pdf>. Acesso em: 11 jun. 2014. 
PISANI, Francis; PIOTET, Dominique. Como a web transforma o mundo: a alquimia das multidões. São Paulo: Senac, 2010.

PROFICIÊNCIA. História do método científico. Disponível em:

<http://www.proficiencia.org.br/article.php3?id_article=489>. Acesso em: 07 jun. 2014.

QUORA. Site. Disponível em: <https://www.quora.com/>. Acesso em: 11 jun. 2014.

SELTZER, Ethan; MAHMOUDI, Dillon. Citizen participation, open innovation and crowdsourcing: challenges and opportunities for planning. Journal of Planning Literature, vol. 28, n. 1, February 2013. Disponível em:

<http://jpl.sagepub.com/content/28/1/3.full.pdf+html>. Acesso em: 10 jun. 2014.

SHIRKY, Clay. A cultura da participação: criatividade e generosidade no mundo conectado. Rio de Janeiro: Zahar, 2011.

SILVA, Edima Aranha. Evolução histórica do método científico: desafios e paradigmas para o século XXI. Econ. Pesqui., Araçatuba, v. 3, n. 3, mar. 2001. Disponível em: 〈http://www.feata.edu.br/downloads/revistas/economiaepesquisa/v3_artigo07_evolucao.pdf> . Acesso em: 12 jun. 2014.

SILVA, Tarcízio. Monitoramento de mídias sociais. In: Para entender o monitoramento de mídias sociais. [Ebook] publicado em 2012. Disponível em: $<$ http://tarciziosilva.com.br/blog/entenda-o-monitoramento-de-midias-sociais-com-e-bookbrasileiro/>. Acesso em: 12 jun. 2014.

VICENTE, Renato. Método científico. Publicado em 2008. Disponível em: <http://www.ime.usp.br/ rvicente/MetodoCientifico.pdf>. Acesso em: 07 jun. 2014.

WAGORN, Paul. The problems with crowdsourcing and how to fix them. Publicado em: 08 abr. 2014. Disponível em: <http://www.ideaconnection.com/blog/2014/04/how-to-fixcrowdsourcing/>. Acesso em: 12 jun. 2014.

WEINBERGER, David. A nova desordem digital: os novos princípios que estão reinventando os negócios, a educação, a política, a ciência e a cultura. Rio de Janeiro: Elsevier, 2007.

WIKIPEDIA. História da Wikipedia. Editado em 23 maio 2014. Disponível em: <http://pt.wikipedia.org/wiki/Hist\%C3\%B3ria_da_Wikip\%C3\%A9dia>. Acesso em: 09 jun. 2014. 
Como citar este documento:

PRADO, Jorge Moisés Kroll do. Crowdsourcing como método de pesquisa: o excedende cognitivo em prol da produção científica.Revista Digital de Biblioteconomia e Ciência da Informação, Campinas, SP, v. 13, n. 2, p. 356-364, maio/ago. 2015. ISSN 1678-765X. Disponível em: 〈http://periodicos.bc.unicamp.br/ojs/index.php/rdbci/article/view/2109>. Acesso em: 31 maio 2015. 